\title{
PARAMETRIC STUDY OF MICROWAVE ABSORPTION IN LOSSY DIELECTRIC SLABS
}

\author{
Isabelle Huynen \\ Institute of Information and Communications Technologies, Electronics and \\ Applied Mathematics (ICTEAM), Université catholique de Louvain, 1348 Louvain- \\ la-Neuve, Belgium
}

\begin{abstract}
This paper presents a thorough study of the behaviour of microwave absorption occurring in lossy dielectric slabs as used for microwave absorbers. The influence of the various parameters governing absorption is demonstrated : dielectric constant, conductivity, thickness of slab and frequency. It is shown that for several combinations of values for these parameters absorption can be maximized to up to unity, i.e. all the incident power is absorbed.
\end{abstract}

\section{KEYWORDS}

microwave absorbers, parametric study.

\section{INTRODUCTION}

The reduction of electromagnetic pollution and electromagnetic interferences is a major technological issue considering the widespread use of wireless applications. The protection of living cells or the preservation of the operation of sensitive electronic devicesconstitute prominent examples. In most applications the unwanted radiation is either confined at the source or the device is shielded by a metallic enclosure that reflects the incident radiation. Most of the time this approach provides cheap and effective solutions. But for some applications shielding based on true absorption of the EM wave with no reflection and no transmission is needed or at least preferred [1]. One example is radar stealth, which consists in reducing the detectability of a target by cancelling reflection of a radar incident to the surface. Usually radar absorbing materials are made of thick foam designed for wideband absorption. []Alternatively, thin multilayered coatings can be used but with operation on a narrow frequency band, except when carbon nanotube nanocomposites are considered [2-4] .Another approach consists in Frequency-Selective Surfaces (FSS) that enable to achieve nearly perfect absorption, however in a narrow frequency band since planar resonators are used to achieve absorption at resonance [5- 6].A further development combines FFS structures with nanocomposite lossy slabs [7].

In this paper we perform a thorough study of the absorption performances of conductive slabs applicable for example to nanocomposite polymer slabs including carbon nanotubes [2-4]. The influence of electrical parameters of the slab, of its-thickness and of the frequency is investigated and explained. 


\section{THEORETICAL FORMULATION OF ELECTROMAGNETIC ABSORPTION}

The absorption in the slab is the fraction of power which is not reflected nor transmitted. The absorption depends on several parameters such as the frequency, the dielectric constant $(\varepsilon \rho)$ and the conductivity $\sigma$ trough the reflection coefficient $\Gamma$ at input

interface of the slab and the transmission factor Tf over sample thickness $\mathrm{d}$ which follows the relations :

$\sqrt{\sqrt{\varepsilon_{e f f}}-1}-1$

$T_{f}=e^{-y a}$

The transmission factor characterizes the propagation inside the slab and its attenuation since $\gamma$ is expressed as

$\gamma=\alpha+j \beta=j \varpi \frac{\sqrt{\varepsilon_{\text {eff }}}}{c_{o}}$

where $\varepsilon f f$ is the effective permittivity which depends on the relations

$\varepsilon_{e f f}=\varepsilon_{r}+\underset{j \pi s \varepsilon_{0}}{\sigma}$

The chain matrix relating input and output voltages associated to electromagnetic waves has the following expression :

$T=\left[\begin{array}{cc}\cosh (\gamma d) & Z_{\varepsilon} \sinh (\gamma d) \\ Z_{c}^{-1} \sinh (\gamma d) & \cosh (\gamma d)\end{array}\right]=\left[\begin{array}{ll}A & B \\ C & D\end{array}\right]$

where $Z_{c}$ is the wave impedance inside the slab given by

$Z_{\tau}=j \frac{w \mu_{O}}{y}=$

The S-parameters are derived from [8]:

$$
\begin{array}{r}
=\frac{A Z_{c}+B-C Z_{c}^{2}-D Z_{c}}{A Z_{c}+B+C Z_{c}^{2}+D Z_{c}} \\
S_{12}=\frac{2(A D-B C) z_{c}^{2}}{A Z_{c}+B-C Z_{c}^{2}+D Z_{c}}
\end{array}
$$

The absorption is finally obtained from the following power balance :

$\mathrm{A}=1-\mathrm{Pr}-\mathrm{Pt}=1-|\mathrm{S} 11|^{2}-|\mathrm{S} 21|^{2}$

according to the definition of S-parameters [9]. 


\section{RESULTS AND DISCUSSION}

The following subsections present the calculation of absorption for several pairs of parameters combining conductivity $\sigma$, dielectric constant $\varepsilon$ r, thickness $d$ of slab $\mathrm{d}$ and frequency.

\section{Conductivity and dielectric constant}

Figure 1 shows the absorption calculated as a function of these two parameters at $10 \mathrm{GHz}$ and for a thickness $d=2 \mathrm{~mm}$. A maximum of absorption is observed for the pair $\left(\varepsilon_{\mathrm{r}}=1, \sigma=3\right)$. This means that for these values a nearly perfect matching occurs between the impedance at input interface of the slab and the impedance of air assuming to surround the slab. For higher values of $\sigma$ attenuation decreases. This is explained by the predominance of $\sigma$ in the reflection coefficient which tends towards unity meaning that all the incident power is reflected, preventing absorption. The same comment holds for $\varepsilon_{r}$ : for high values it dominates in reflection coefficient $\Gamma$ (1) which tends to unity, preventing absorption. As a consequence the reflection at the input of the slab increases as well (Fig 2). The fact that transmission never reaches unity is due to a part of the power being transmitted through the slab to its output interface and air. The same comment is valid for results presented in the next subsections.

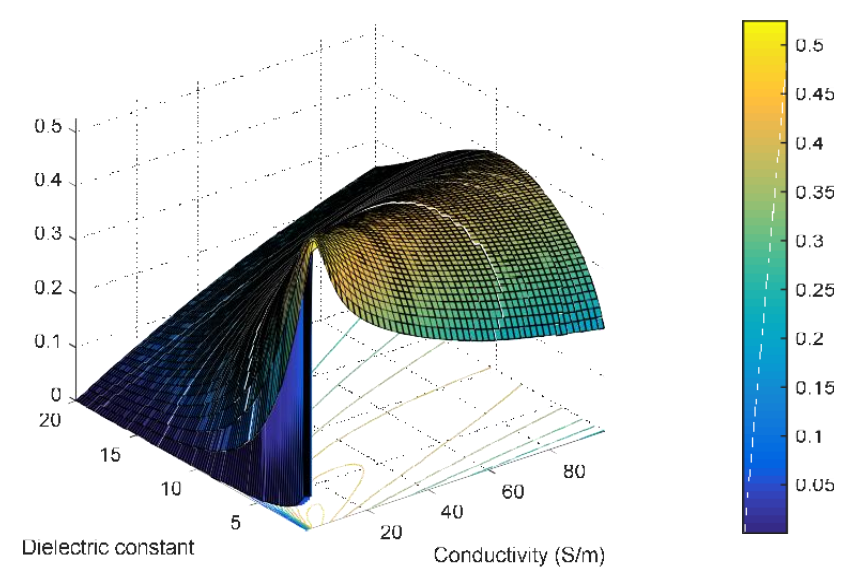

Figure 1. Absorption at $10 \mathrm{GHz}$ as function of conductivity and dielectric constant, for thickness $\mathrm{d}=2$ $\mathrm{mm}$. Maximal absorption occurs for $\varepsilon_{\mathrm{r}}=1$ and $\sigma=3 \Sigma / \mathrm{m}$, and corresponds to a minimal reflection in

Figure 2 .

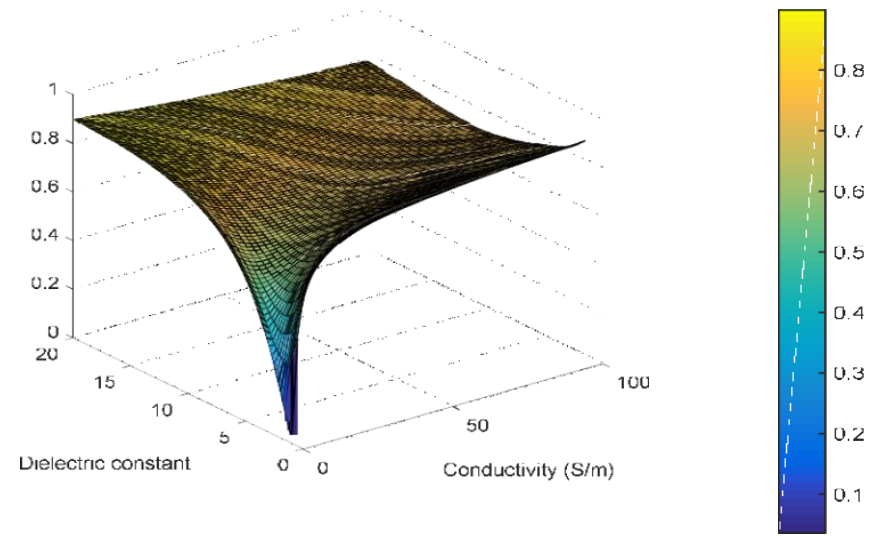

Figure 2. Reflection at $10 \mathrm{GHz}$ as a function of conductivity and dielectric constant, for a thickness $\mathrm{d}$ $=2 \mathrm{~mm}$. Minimal reflection occurs for $\varepsilon_{\mathrm{r}}=1$ and $\sigma=3 \Sigma / \mathrm{m}$, and corresponds to a maximal absorption in Figure 1. 


\section{Conductivity and frequency}

Simulations of Fig. 3 show an increase of absorption with frequency, due to the increase of complex propagation constant with frequency, especially the attenuation factor $\alpha$, responsible for the decrease of transmission factor $\mathrm{Tf}$ inducing absorption according to (2). As in previous section absorption is maximum for the same value of conductivity $(\sigma=3)$ and then decreases for the reason provided in previous section. The dual behavior is observed for the reflection (Fig.4) : it decreases with frequency and increases for high values of conductivity that induces reflection as in section 3.1.

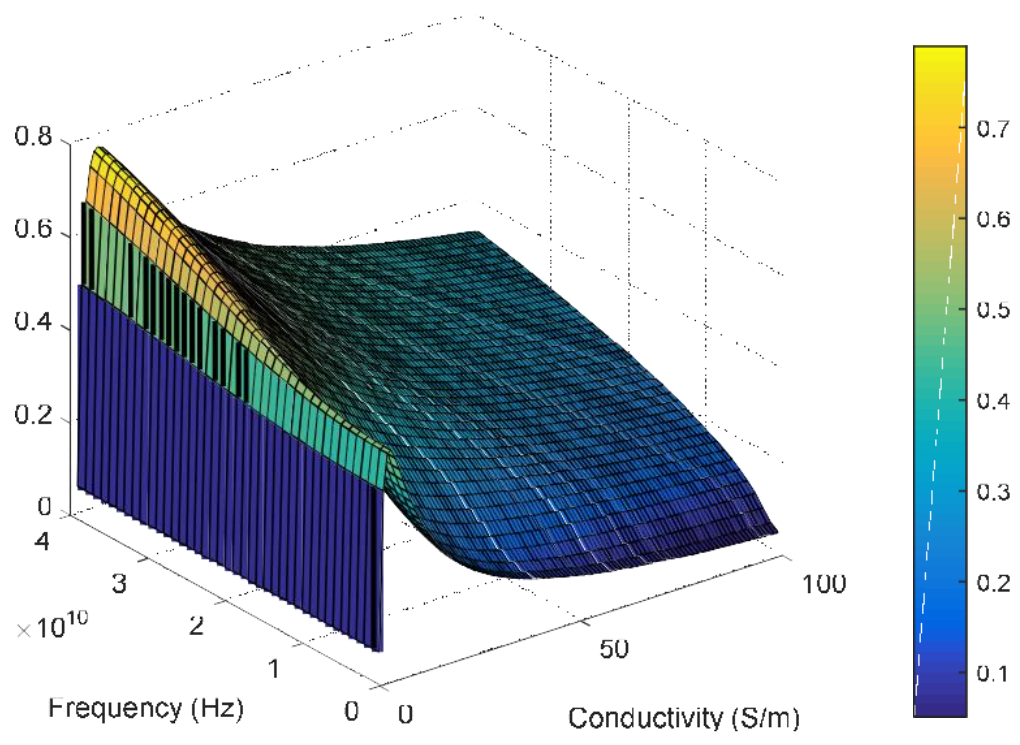

Figure 3. Absorption as function of conductivity and frequency, for a thickness $\mathrm{d}=2 \mathrm{~mm}$ and dielectric constant $\varepsilon_{\mathrm{r}}=1$. Maxima of absorption occur for $\mathrm{s}=3 \Sigma / \mathrm{m}$, in agreement with observation in Figure 1.

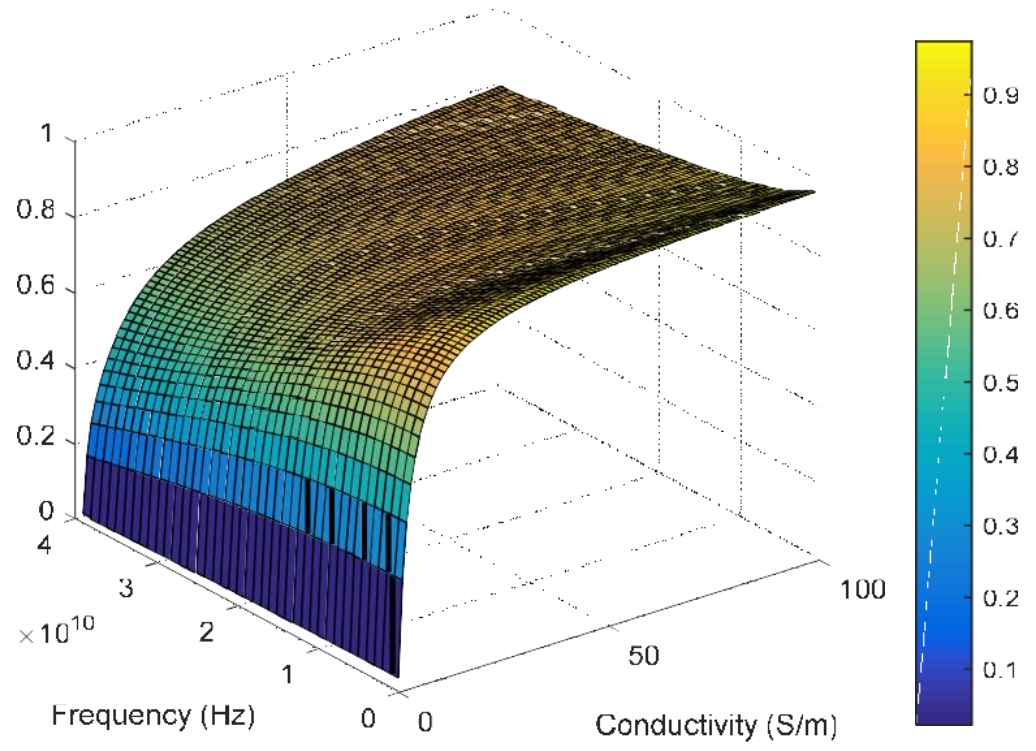

Figure 4. Reflection as a function of conductivity and frequency, for a thickness $\mathrm{d}=2 \mathrm{~mm}$ and dielectric constant $\varepsilon r=1$. The behaviour is dual of that of absorption in Figure 3. 


\section{Absorption as a function of conductivity and thickness}

The increase of absorption with thickness observed in Fig. 5 is correlated to the decrease of transmission factor (2): its exponential factor $\alpha$ (3) is proportional to the thickness which induces attenuation associated to absorption. Absorption tends to saturate for sufficient thickness, i.e. when the product ( $\alpha \times \mathrm{d}$ ) becomes sufficient to extinct the transmission factor $\mathrm{Tf}$ (2). The dependence on conductivity shows a decrease with frequency due to its impact on reflection coefficient as previously explained except for very low thickness where absorption increases with conductivity. This behaviour remains unexplained to date. Reflection in Fig .6 presents as expected the complementary behaviour of absorption (minimum when absorption is maximum and vice versa).

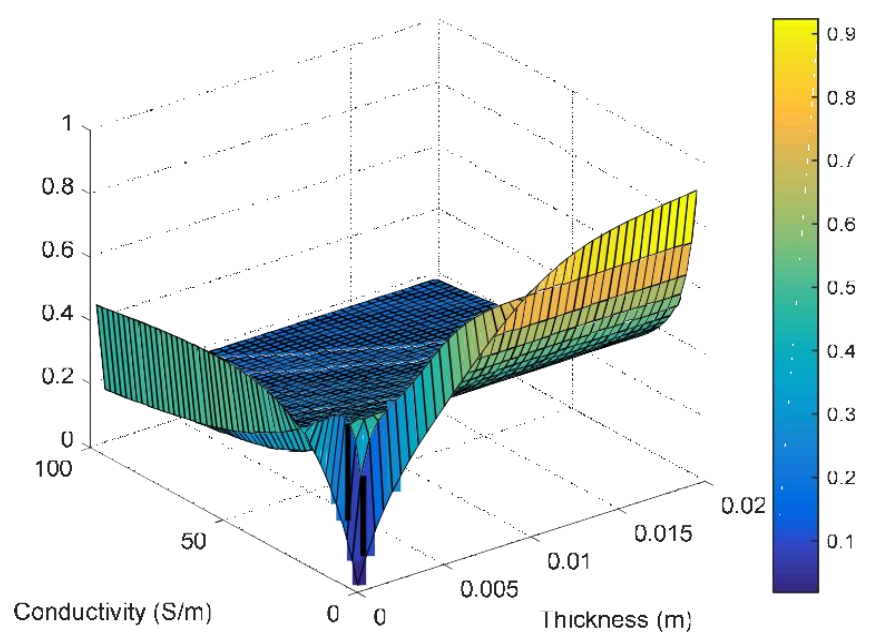

Figure 5 Absorption at $10 \mathrm{GHz}$ as a function of thickness and conductivity for a thickness $\mathrm{d}=2 \mathrm{~mm}$ and dielectric constant $\varepsilon_{\mathrm{r}}=1$.

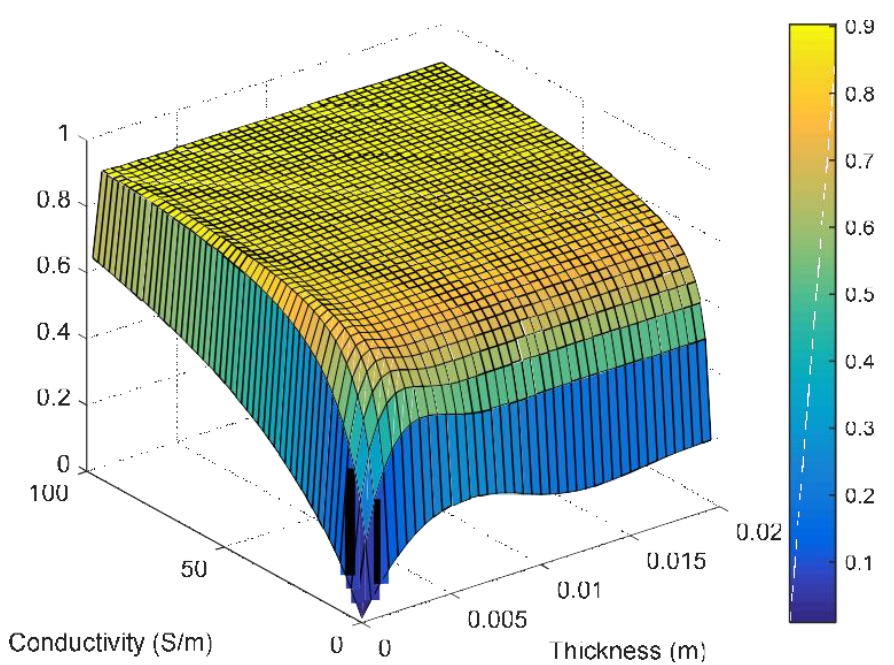

Figure 6. Reflection at $10 \mathrm{GHz}$ as a function of thickness and conductivity for a thickness $\mathrm{d}=2 \mathrm{~mm}$ and dielectric constant $\varepsilon r=1$. The behaviour is dual of that of absorption in Figure 5. 


\section{Dielectric constant ad thickness}

Absorption as shown in Fig. 7 saturates at high thicknesses as observed and explained in the previous section. Above a threshold thickness the transmission factor is vanishes so that no more absorption occurs. The dependence on dielectric constant is as previously observed and explained in section 3.1: its increase increases the reflection coefficient, blocking transmission through the slab, hence the absorption. For high values of dielectric constant some ripple occurs as function of the thickness and is associated to multiple paths of the signal which undergoes successive reflections at input and output interfaces of the slab. The periodicity is short since the wavelength decreases with the dielectric constant and the periodicity associated to multiple reflections is equal to half a wavelength. As in previous subsection the refection in Fig .8 shows a dual behavior.

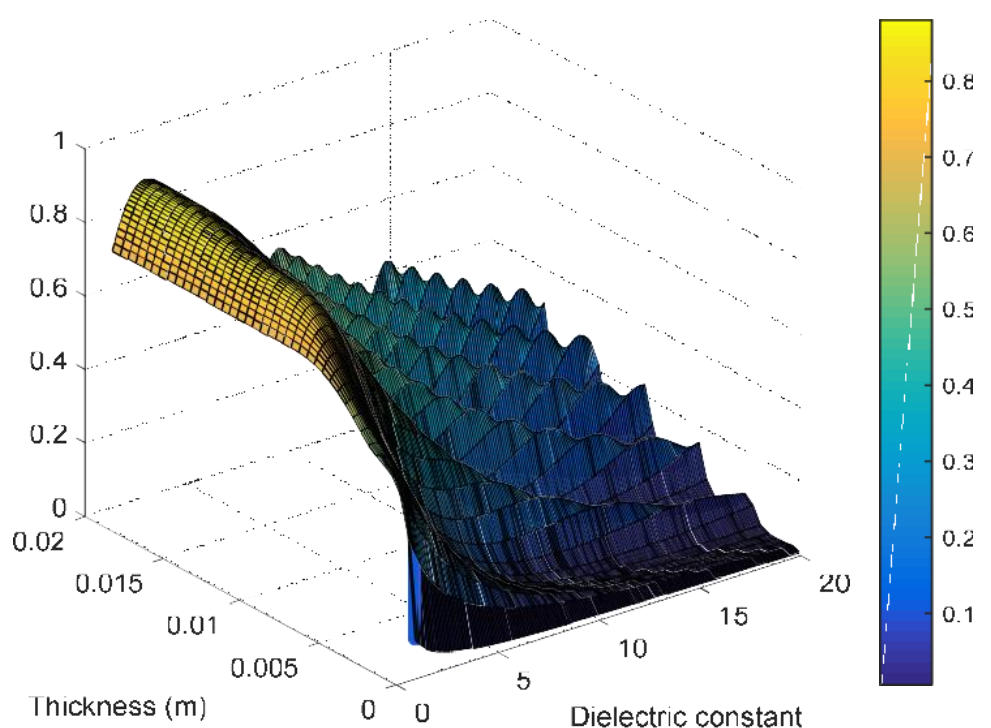

Figure 7. Absorption at $10 \mathrm{GHz}$ as function of dielectric constant and thickness of slab for a conductivity $\sigma=3 \mathrm{~S} / \mathrm{m}$.

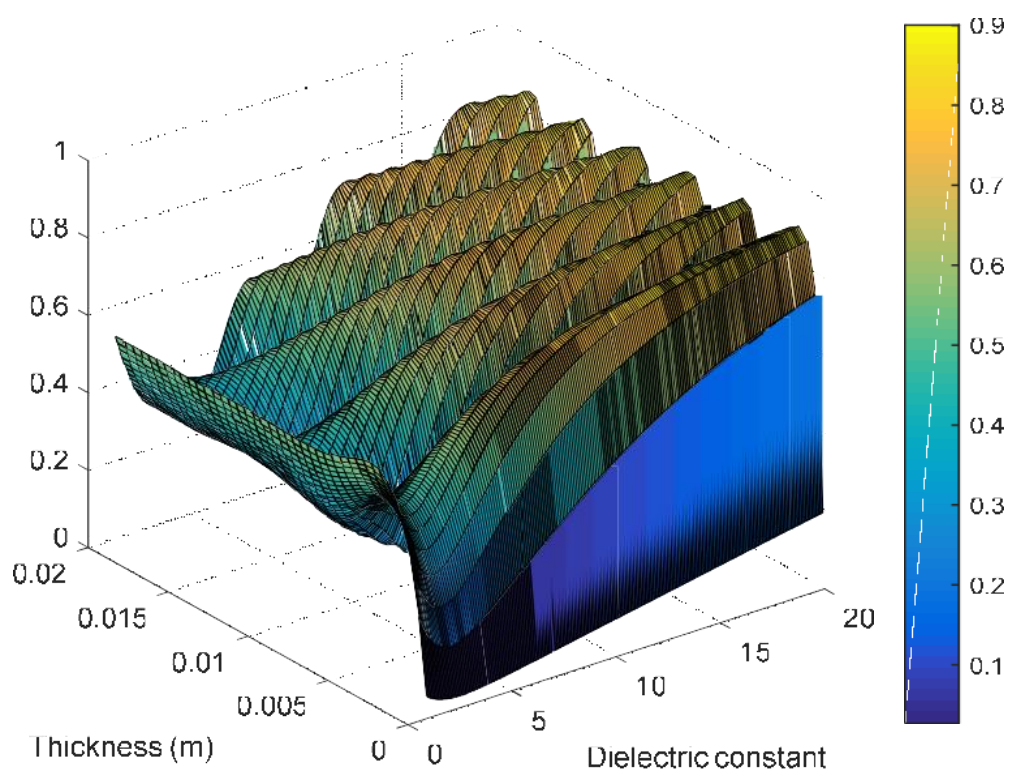

Figure 8. Reflection at $10 \mathrm{GHz}$ as function of dielectric constant and thickness of slab for a conductivity $\sigma=3 \mathrm{~S} / \mathrm{m}$. The behaviour is dual of that of absorption in Figure 7. 


\section{Dielectric constant and frequency}

Absorption as shown in Fig. 9 increases with frequency because the magnitude of the transmission factor $\mathrm{Tf}(2)$ decreases since it is proportional to the product ( $\sigma \mathrm{x} \mathrm{f})$. Absorption decreases with dielectric constant due to increase of reflection coefficient as explained before. Refection shown in Fig. 10 exhibits a dual behaviour.

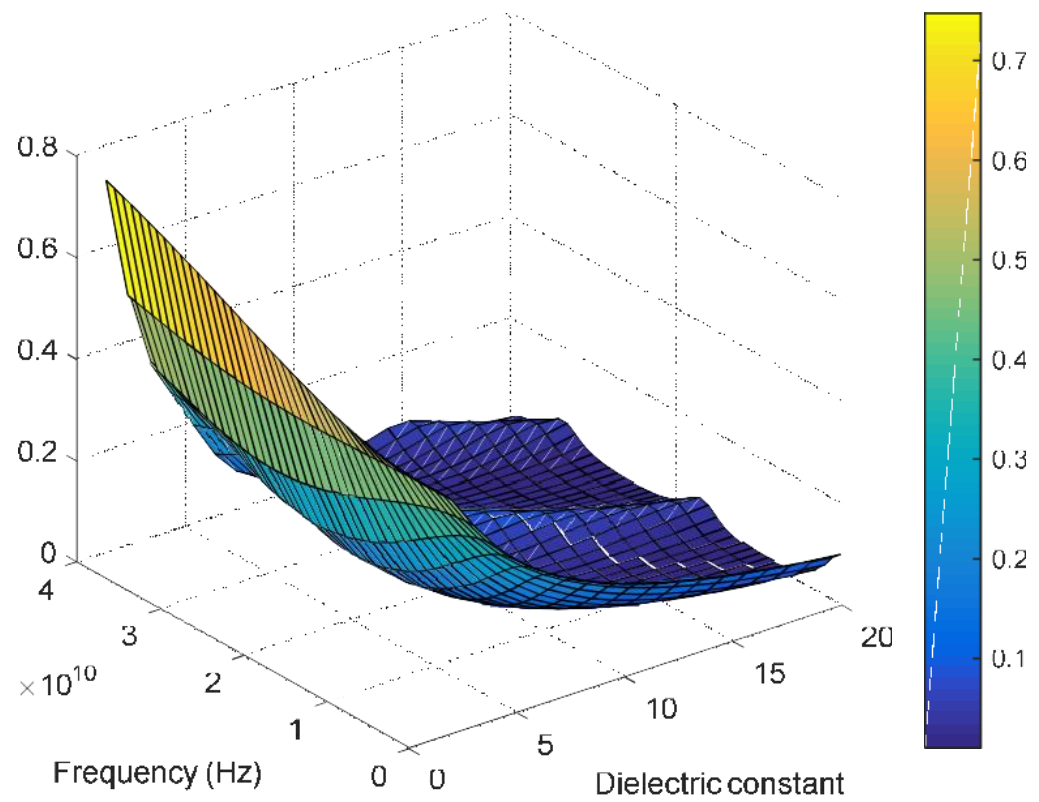

Figure 9. Absorption function of dielectric constant and frequency for a thickness $\mathrm{d}=2 \mathrm{~mm}$ and a conductivity $\sigma=3 \Sigma / \mathrm{m}$.

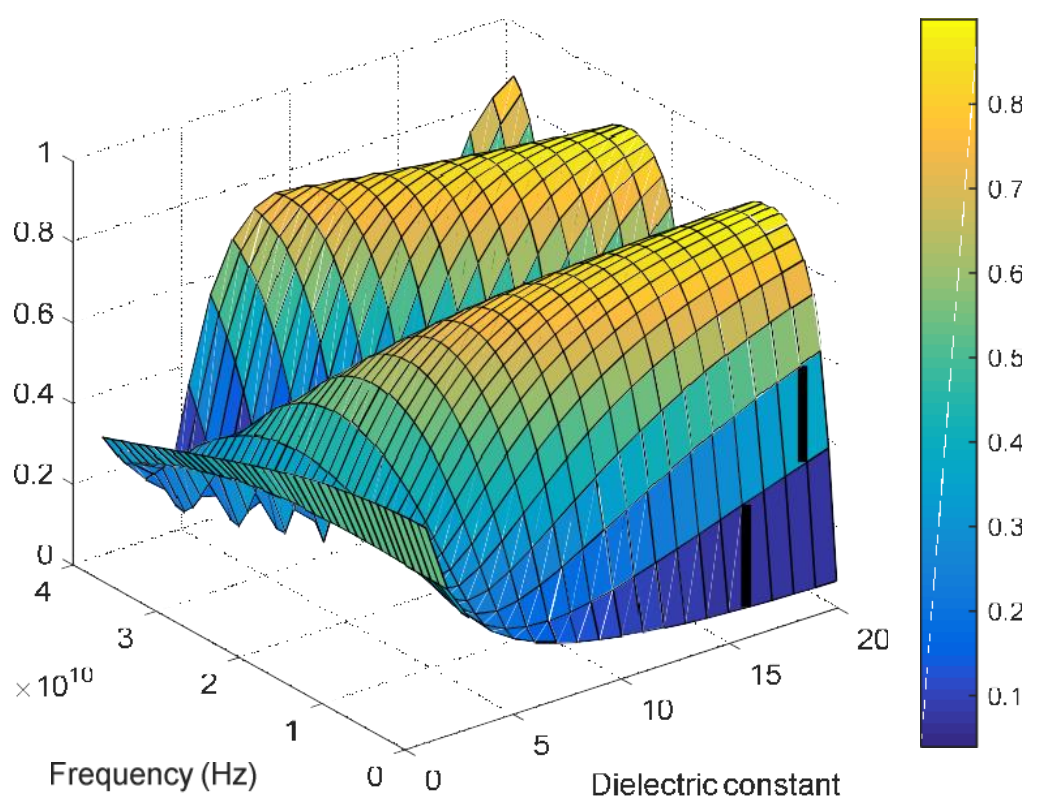

Figure 10. Reflection function of dielectric constant and frequency for a thickness $\mathrm{d}=2 \mathrm{~mm}$ and a conductivity $\sigma=3 \Sigma / \mathrm{m}$. The behaviour is dual of that of absorption in Figure 9. 


\section{Thickness and frequency}

Absorption as shown in Fig. 11 increases as a function of frequency as explained before and is constant whatever the thickness for high frequency. The reason is similar as previously described : above a given thickness the product ( $\mathrm{d} \mathrm{x}$ frequency) is sufficient to extinct the transmission factor. The dependence on thickness for low frequencies remains unexplained. Again the refection shown in Fig. 12 exhibits a dual behavior.

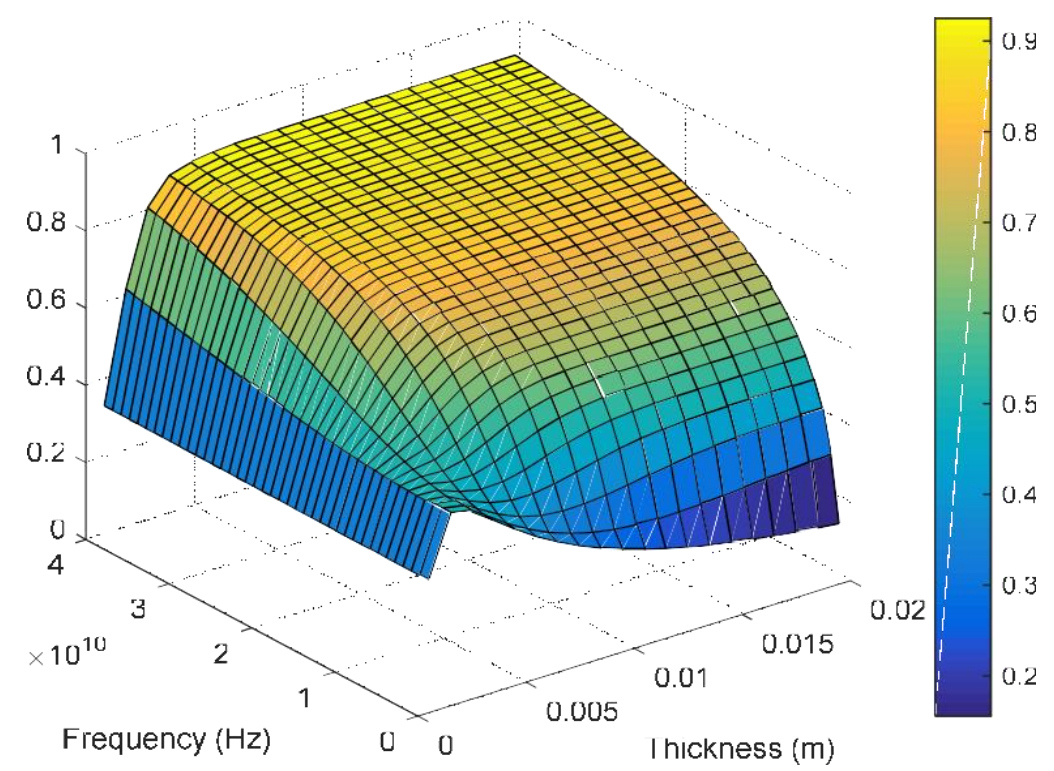

Figure 11. Absorption function of thickness and frequency for a dielectric constant $\varepsilon r=1$ and a conductivity $\sigma=3 \Sigma / \mathrm{m}$.

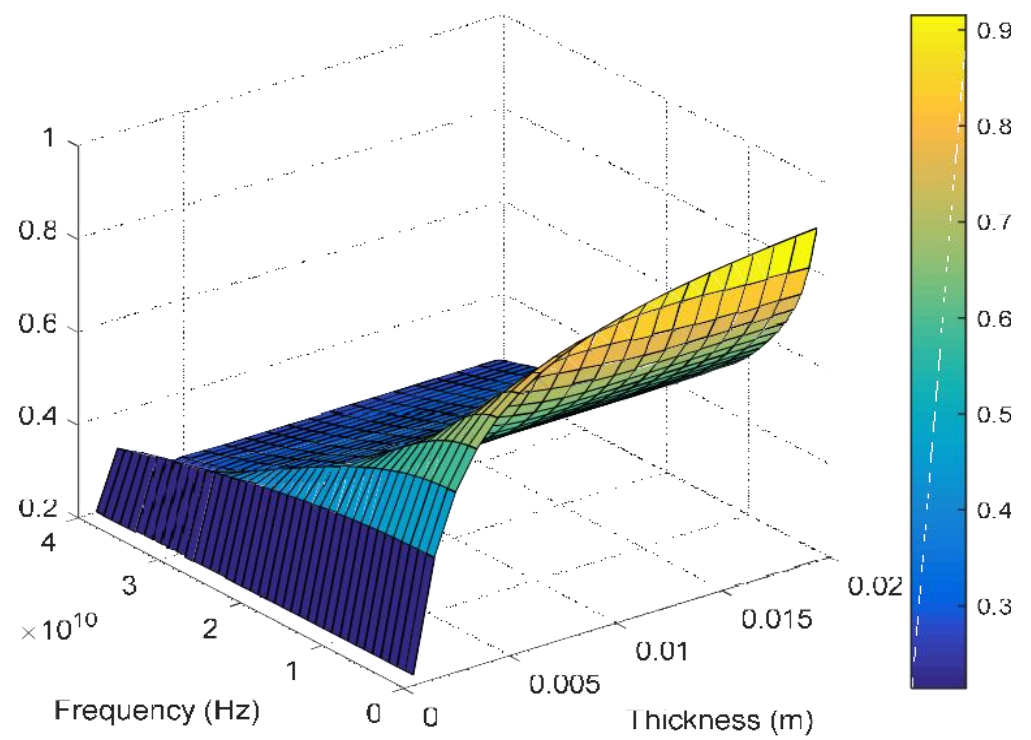

Figure 12. Reflection function of thickness and frequency for a dielectric constant $\varepsilon_{\mathrm{r}}=1$ and a conductivity $\sigma=3 \Sigma / \mathrm{m}$. The behaviour is dual of that of absorption in Figure 11. 


\section{DEPENDENCE OF MAXIMAL ABSORPTION ON PARAMETERS D, $\varepsilon$ P, $\sigma$, AND FREQUENCY}

In section 3.1 absorption has been shown to present a maximum for a specific couple of values ( $\varepsilon r=1$ and $\sigma=3$ ). The observation was made for a frequency of $10 \mathrm{GHz}$ and a thickness d equal to $\mathrm{d} 2 \mathrm{~mm}$. In this section the dependence on thickness and frequency of the couple of values $\mathrm{\varepsilon r}$ and $\sigma$ providing maximal absorption is studied, as well as the magnitude of absorption. At 10 $\mathrm{GHz}$, for each thickness the values of $\varepsilon r$ and $\sigma$ providing maximal absorption are determined in section 4.1. The same is done ass a function of frequency for a thickness $\mathrm{d}=2 \mathrm{~mm}$ in section 4.2.

\section{Absorption versus thickness}

The frequency is assumed equal to $10 \mathrm{GHz}$. As expected absorption increases in Fig.13 with thickness, due to its impact on the transmission factor $\mathrm{Tf}$ (2). The value of dielectric constant providing maxima absorption does not change with thickness (Figure 13).: it remains equal to 1 in order to minimize reflection of the wave at input interface of the slab. However the conductivity decreases with thickness. This can be explained by the influence of attenuation coefficient a, proportional to the product ( $\sigma \times \mathrm{d}$ ), so that for a given level of absorption, of equivalently of attenuation factor, the conductivity decreases with thickness.

\section{Absorption versus frequency}

As expected absorption increases with frequency (Fig. 15), due to its impact on the transmission factor $\mathrm{Tf}$ (2). The value of dielectric constant providing maxima absorption does not change with thickness while the conductivity increases with frequency, as shown in Fig. 16. The conductivity $\sigma$ increases with frequencysince absorption follows the extinction of transmission factor $\mathrm{Tf}$ which is proportional to $\sigma$.

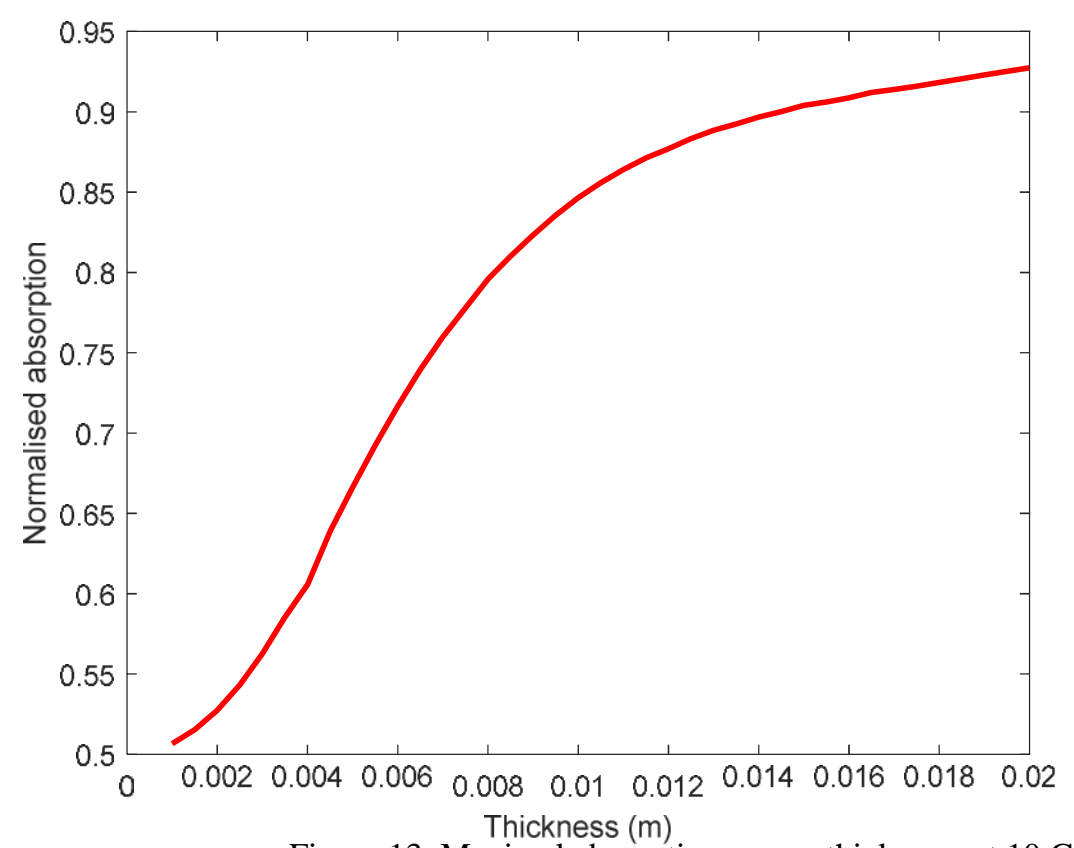

Figure 13. Maximal absorption versus thickness, at $10 \mathrm{GHz}$. 
International Journal Of Microwave Engineering (JMICRO) Vol.1, No.4, October 2016

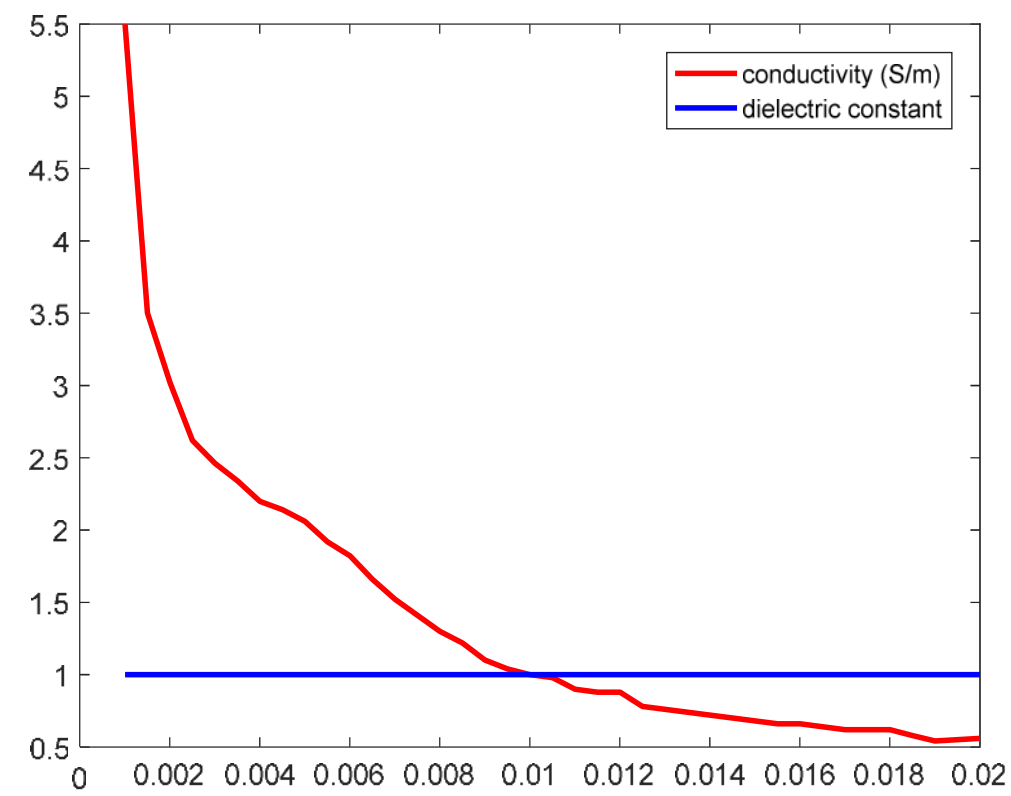

Figure 14. Dielectric constant and conductivity providing maximal absorption at $10 \mathrm{GHz}$.

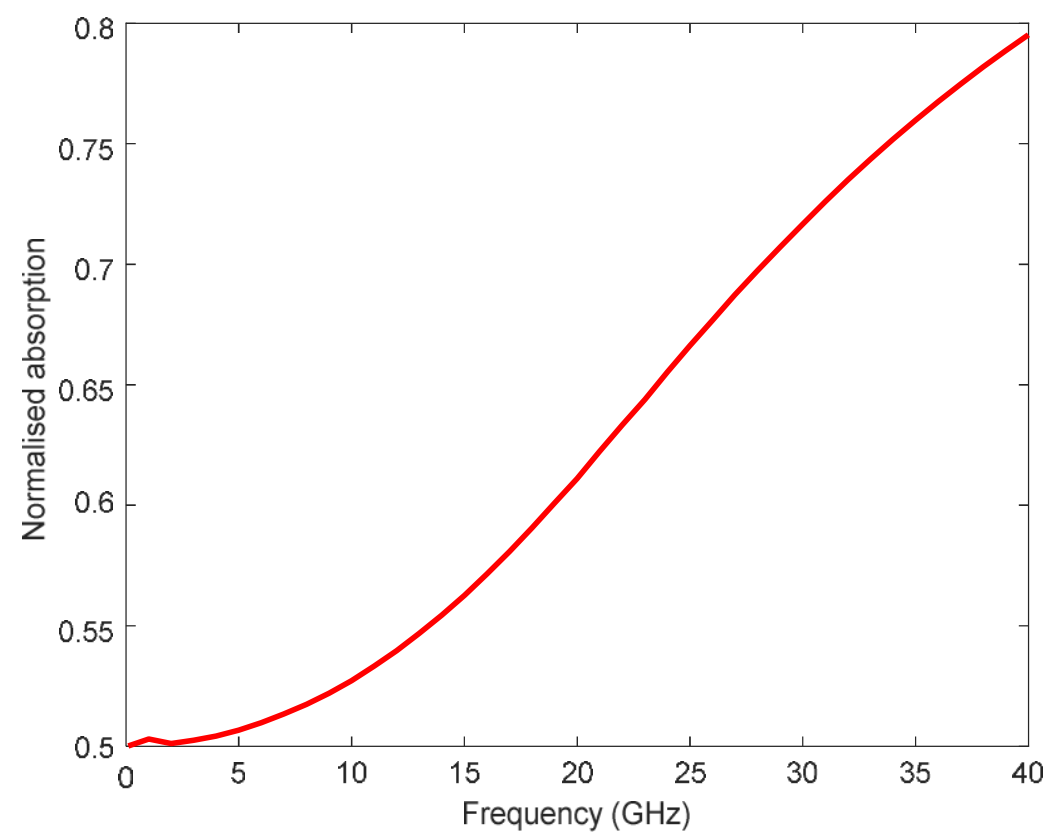

Figure 15. Maximal absorption as function of frequency, for $\mathrm{d}=2 \mathrm{~mm}$. 


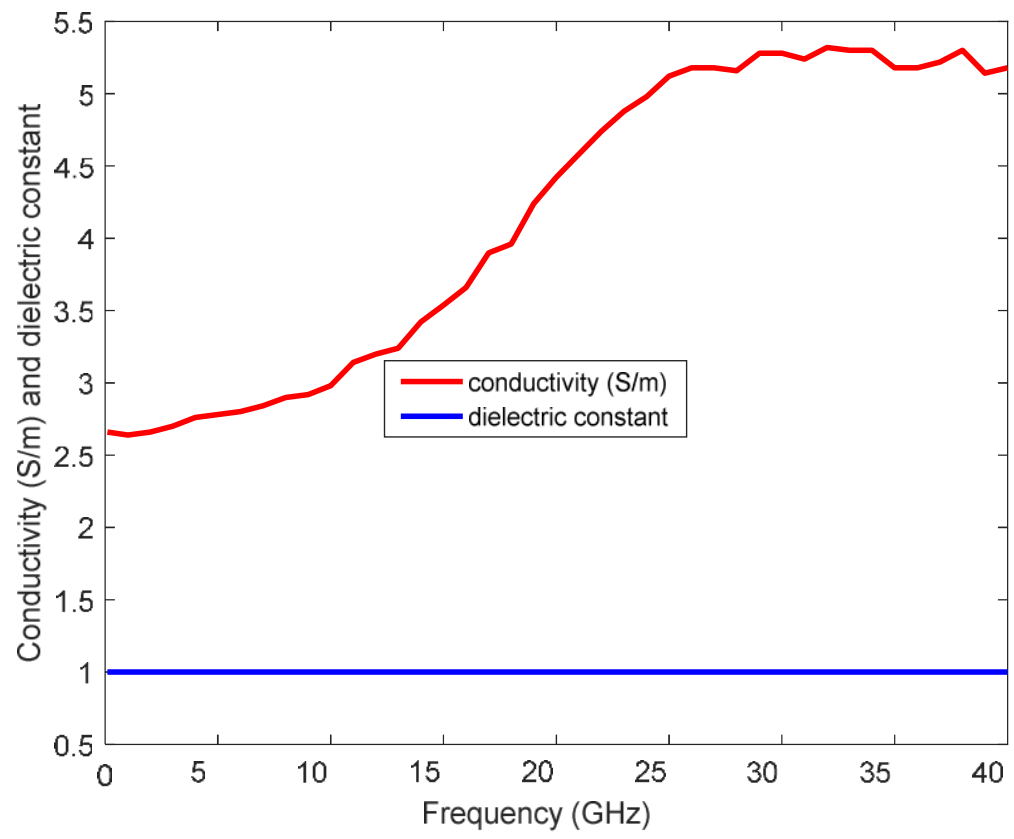

Figure 16. Dielectric constant and conductivity providing maximal absorption for $\mathrm{d}=2 \mathrm{~mm}$, as function of frequency.

\section{CONCLUSION}

We presented a thorough study of the behaviour of microwave absorption occurring in lossy dielectric slabs as used for microwave absorbers. The influence of the various parameters governing absorption is demonstrated: dielectric constant, conductivity, thickness of slab and frequency. At fixed thickness of the slab it is shown that for particular combinations of values for these parameters absorption can be maximized i.e. when the dielectric constant er is equal to 1 . In this case the reflection of incident wave at input interface of the slab is minimized. Then for sufficient value of the conductivity $\sigma(5.4 \mathrm{~S} / \mathrm{m})$ absorption reaches up to $80 \%$ at $40 \mathrm{GHz}$. On the other hand for sufficient thicknesses of the slab nearly the all incident power is absorbed. (95\%). This study is helpful for understanding the mechanisms of absorption in lossy dielectric slabs and can be used for the design of FSS absorbers.

\section{ACKNOWLEDGMENTS}

The author is grateful to the National Fund for Scientific Research (F.R.S.-FNRS) and FRIA for supporting this research. This work is also supported by the Walloon region through the projects Multimasec and Atac-Concept, and by the Communauté Française de Belgique, through the project "Nano4waves" funded by its research program "Actions de Recherche Concertées". The author thanks P. Bollen, Y. Danlée, C Bailly and T. Pardoen for fruitful discussions. 


\section{REFERENCES}

1. Xingcun Colin Tong, "Advanced Materials and Design for Electromagnetic Interference Shielding”, CRC Press, November 19, 2008.

2. Yann Danlée, Isabelle. Huynen, Christian Bailly, «"Thin smart multilayer microwave absorber based on hybrid structure of polymer and carbon nanotubes", Applied Physics Letters, vol. 100, no. 21, 2012, pp.213105.1-3.

3. Yann Danlée, Christian Bailly, Isabelle Huynen, "Thin and flexible multilayer polymer composite structures for effective control of microwave electromagnetic absorption", Composites Science and Technology, vol. 100, August 2014, pp. 182-188.

4. Nicolas Quiévy, Pierre Bollen, Jean-Michel Thomassin, Christophe Detrembleur, Thomas Pardoen, Christian Bailly and Isabelle Huynen, "Electromagnetic absorption properties of carbon nanotube nanocomposite foam filling honeycomb waveguide structures", IEEE Transactions on Electromagnetic Compatibility (Special Issue on Nanotechnology), vol 54, no.1, February 2012, pp 43-51.

5. Jinpil Tak, Yunnan Jin, and Jaehoon Cho, “A dual band metamaterial microwave absorber", Microwave and Optical Technology Letters, vol. 58, no. 99, June 2016, pp. 2052-2055.

6. Meng Zhang,,Tian, Jiang, Yijun Feng, "Design and Measurement Of Microwave Absorbers Comprising Resistive Frequency Selective Surfaces”, Journal of Electromagnetic Analysis and Applications, 2014, vol. 6, no. 6, pp. 203-208.

7. Rajkumar Jaiswar, Yann Danlée, Henok Mesfin, Arnaud Delcorte, Sophie Hermans, Christian Bailly, Isabelle Huynen, "Absorption Modulation of FSS-Polymer nanocomposites through Incorporation of Graded Conductive nano-fillers", Proceedings of the META 2016 Conference, Malaga, Spain, 25-28 June, 6 pp.

8. Janusz.A. Dobrowolski, Introduction to computer method for microwave circuit analysis and design, Artech House, 1991.

9. David Pozar, Microwave engineering, 4th edition, 2012.

\section{Authors}

Isabelle Huynen was born in 1965 in Brussels, Belgium. She earned the PhD degree in Applied Sciences in 1994 at the Université catholique de Louvain (U.C.L). Since 1999 she is Research Director of the National Fund of Scientific Research and part-time Professor at UCL. 